\title{
Gender discrimination in algorithmic decision-making
}

\author{
Andreeva, Galina ${ }^{a}$ and Matuszyk, Anna ${ }^{\text {b,c }}$
}

${ }^{a}$ Business School, University of Edinburgh, UK, ${ }^{\text {b }}$ Stern Business School, New York University, USA, ${ }^{\mathrm{c}}$ Warsaw School of Economics, Poland.

\begin{abstract}
Most countries prohibit the use of Gender when deciding whether to give credit to prospective borrowers or not. The increasing application of automated algorithmic-based decision-making raises series of questions as to how the discrimination may arise and how it can be avoided. In this paper we analyse a unique proprietary dataset on car loans from an EU bank with the objective to understand if the minority status of females amplifies gender bias, and if there are ways to mitigate it. The initial results show that Gender is statistically significant, and women show lower probability of default. However, if Gender is excluded from the model, women have lower chances to be accepted for credit as compared to the situation when it is included. Women constitute only a quarter of the sample, and we investigate if this may lead to a representation bias which could amplify the discrimination. We experiment with under- and over-sampling and explore the effect of balancing the training set on mitigating discrimination. Logistic regression is used as a benchmark with further plans to include random forests. The results are applicable to other situations where predictive models based on historical data are used for decision-making. The presentation will discuss initial results and work in progress.
\end{abstract}

Keywords: credit scoring; gender disrimination; algorithmic decisionmaking. 